\title{
Screening obstructive sleep apnoea syndrome by home videotape recording in children
}

\author{
Y. Sivan*, A. Kornecki*, T. Schonfeld**
}

Screening obstructive sleep apnoea syndrome by home videotape recording in children. Y. Sivan, A. Kornecki, T. Schonfeld. (CERS Journals Ltd 1996.

ABSTRACT: Overnight polysomnography (PSG) has been used to diagnose and assess the severity of obstructive sleep apnoea syndrome (OSAS) in children. The aim of this study was to determine whether home video-recording of children during sleep may be used for screening OSAS.

In 58 children suspected of having OSAS, PSG results were compared with the corresponding analyses of $\mathbf{3 0}$ min video-recordings of each child sleeping at home. The video-recordings were evaluated by: 1) overall investigator's clinical judgement; and 2) a scoring system based on noisy breathing, movements, waking episodes, apnoea, chest retractions and mouth breathing.

PSG results were highly correlated with the video test results, with agreement in 49 out of $58(84 \%)$. In 36 of the 58 children, the PSG was abnormal compared with 41 out of 58 abnormal video tests. The sensitivity of the overall investigator judgement of video test was $94 \%$ ( 34 out of 36 ) and the specificity $68 \%$ (15 out of 22 ). Video scores $>10$ were highly predictive of OSAS, whilst scores $<5$ were associated with normality. Using a stepwise logistic regression analysis, each of the scoring variables was tested against the PSG results and an equation was formulated for predicting PSG by the video test. The equation predicted PSG results in 49 out of $58(84 \%)$ cases.

Thirty minutes of home video-recordings during sleep is a reliable screening method for obstructive sleep apnoea syndrome in children. This technique may, thus, improve patient selection for polysomnography.

Eur Respir J., 1996, 9, 2127-2131.
*Pediatric ICUs, Pulmonary Clinics and Sleep Laboratory, Dana Children's Hospital, Tel-Aviv Sourasky Medical Center, and **Shneider Children's Medical Center, TelAviv University, Sackler Faculty of Medicine, Israel.

Correspondence: Y. Sivan

Pediatric ICU

Dana Children's Hospital

Tel-Aviv Sourasky Medical Center

6 Weizman Street

Tel-Aviv 64239

Israel

Keywords: Adenotonsillar hypertrophy children

obstructive sleep apnoea syndrome polygraphic sleep study

snoring

video-recording

Received: December 121995

Accepted after revision June 271996
Obstructive sleep apnoea syndrome (OSAS) in children is a disorder of breathing during sleep characterized by prolonged partial upper airway obstruction and/or intermittent complete obstruction that disrupts normal ventilation during sleep and normal sleep patterns [1-3]. Patients and their parents usually consult the paediatrician or the ear, nose and throat (ENT) physician because of noisy breathing, snoring, cessation of breathing, increased body movements and awakenings during sleep [2, 3]. However, most children with OSAS are examined by physicians during wakefulness, whilst breathing normally, when the signs and symptoms of OSAS are not usually apparent $[4,5]$. However, these children may have severe problems in breathing whilst asleep $[6,7]$.

Polygraphic sleep study (polysomnography (PSG)) is an objective and noninvasive test for assessing the OSAS and its severity $[1,8]$. However, PSG is expensive and results may be affected by environmental and instrumentation effects.

In order to observe children whilst asleep in their natural environment, home video-recording may be used. This may improve physicians understanding of the child's breathing problem and add important information to the physical examination. If home video-recording of the sleeping child is found to be reliable and sensitive in diagnosing OSAS, it may then be used also for screening patients with clinically significant upper airway obstruction (UAO). In a recent textbook of paediatric respiratory diseases [4], home video-recording during sleep has been suggested as the first step in the clinical evaluation of children with suspected OSAS, and also as a screening method. Nevertheless, at present, no studies comparing home video-recording to laboratory PSG have been reported

The purpose of this study was to compare the results of PSG in children suspected of having OSAS with the observation of these children during sleep at home using a video-recording, and to investigate whether home videorecording of the sleeping child may be used for screening OSAS.

\section{Patients and methods}

Fifty eight consecutive children whose parents consulted their paediatrician or ENT physician for snoring or laboured breathing during sleep (nocturnal sweating, special sleeping positions, restless sleep, awakening and excessive movements) were included in the study. All children had enlarged adenoids, or adenoids and tonsils, 
which were confirmed by ENT examination. This was also confirmed in all cases by lateral radiography of the nasopharynx using a copper filter to enhance airway contour and soft tissue outline. The children were aged 2-6 yrs (median 4.3 yrs). No children with other causes of UAO or other coexisting conditions were referred during the study period.

All parents recorded their child on a videotape cassette during night-time sleep at home. The recording consisted of a close-up exposure of the head and naked trunk of the child for $30 \mathrm{~min}$ of sleep. Parents were instructed to record their child during periods of snoring, laboured breathing, or when they considered that their child had a breathing problem while asleep (worst breathing). All parents were instructed to prevent background noise, including noise from television sets and loud conversations, both in the patient's and adjacent rooms. The videorecordings were evaluated by: 1) a scoring system where points were assigned to each variable (table 1); and 2) overall investigator's subjective interpretation and judgement of sleep as being either normal or abnormal (OSAS).

All children underwent overnight PSG for at least $8 \mathrm{~h}$. The following variables were continuously measured and recorded: heart rate and electrocardiographic activity (ECG); respiratory movements and breathing rate by thoracic impedance; arterial oxygen saturation $\left(\mathrm{Sa}, \mathrm{O}_{2}\right)$ by pulse oximetry; oximeter pulse wave form; and airflow and end-tidal carbon dioxide tension $\left(P \mathrm{ET}, \mathrm{CO}_{2}\right)$ by capnography. $P$ ET, $\mathrm{CO}_{2}$ values were accepted only when a $\mathrm{CO}_{2}$ plateau was present. Sleep behaviour, respiratory events, the presence of chest retractions and respiratory pauses were recorded.

PSGs were defined as being abnormal in the presence of one or more of the following: 1) an average of more than one obstructive apnoea of any length per hour of sleep; 2) any episode of obstructive apnoea associated with hypoxaemia $\left(\mathrm{Sa}_{\mathrm{a}, \mathrm{O}_{2}}<90 \%\right.$ or a decrease greater than $4 \%$ from baseline $\left.\mathrm{Sa}_{\mathrm{a}} \mathrm{O}_{2}\right) ; 3$ ) central apnoea $>20 \mathrm{~s}$ or associated with desaturation; 4) hypoventilation (peak $P$ ET, $\mathrm{CO}_{2}$

Table 1. - Video-recording test scoring system

1. Inspiratory noise:

0 - None

1 - Weak

2 - Loud

2. Type of inspiratory noise:

1 - Episodic

2 - Continuous

3. Movements during sleep:

0 - No movements

1 - Few movements $(\leq 3)$

2 - Numerous movements $(\geq 3)$, whole body

4. Number of waking episodes:

1 point for each episode

5. Number of apnoeas:

0 - None

1 - One or two

2 - Numerous; $\geq 3$

6. Chest retractions:

0 - None

1 - Intermittent (periodic)

2 - All the time

7. Mouth breathing:

0 - None

1 - Intermittent (periodic)

2 - All the time $\geq 7.1 \mathrm{kPa}\left(53 \mathrm{mmHg}\right.$ ), or $P$ ET, $\mathrm{CO}_{2} \geq 6.0 \mathrm{kPa}$ (45 mmHg) for more than $60 \%$ of total sleep time or associated with hypoxaemia as above, or a change in $P \mathrm{ET}, \mathrm{CO}_{2}>1.7 \mathrm{kPa}$ (13 $\mathrm{mmHg}$ ) from baseline) [4, 9-12].

For every patient, the PSGs and the video studies were analysed separately, each by a different investigator unaware of the results of the other study: for 29 children the PSGs were evaluated by investigator No. I and the corresponding video recordings by investigator No. II; and for the other 29 children, investigator No. I reviewed the video-recordings while investigator No. II evaluated the corresponding PSGs. In addition, the video-recordings were assessed by a third investigator and the interinvestigator variability of both overall interpretation and scores was studied. All three investigators were paediatric pulmonologists familiar with sleep-related breathing disorders and with considerable experience with OSA. In addition, 20 of the 58 video-recordings were also analysed by another clinician, a consultant paediatrician with no special training in paediatric pulmonology (hereafter termed "observer"). The observer analysed the 20 video-recordings as above, after being trained for $45 \mathrm{~min}$ by the investigators. The observer/investigators difference was analysed. Home sleep video-recording was always performed within 4 days of the PSG.

The results of the video-recordings were compared with the PSGs. In order to evaluate the prediction of the PSG results using the scoring system, a stepwise logistic regression was applied using all seven score variables [13]. Individual parameters used for scoring the videorecordings were examined against the findings of PSG. The association between the video test results and the PSG results was assessed by the Fisher's exact test. Kappa test of reliability was used to assess the degree to which the PSG results were represented by the video test. The sensitivity and the specificity of the video test in relation to the PSG were calculated. Statistical analysis was performed by the Statistical Laboratory, Tel Aviv University. A p-value of $<0.05$ was considered significant.

\section{Results}

The two investigators who analysed the video-recordings agreed in their overall interpretations in 56 out of 58 cases $(97 \%)$. The two cases of disagreement were judged as "inconclusive" by one investigator and as "normal" by the other. A difference in video score of one point between the two investigators occurred in 3 of the 58 cases $(5 \%)$ (the lower score was elected), and a difference of two points in one case $(2 \%)$ (the mean score was elected). These disagreements included: "number of apnoeas" (two disagreements); "type of inspiratory noise" and "movements during sleep and number of waking episodes" (one disagreement each). The observer agreed with the investigators' overall interpretation in 18 out of 20 recordings $(90 \%)$ and disagreed in 2 out of 20 . An observer/investigators score difference of one point occurred in two cases $(10 \%)$, and of two points in one case. In two of these three disagreements the observer's score was higher than the investigator's.

PSG was abnormal in 36 of the 58 children (62\%) compared with 41 of the 58 abnormal video-recordings (71\%) assessed by the overall investigator interpretation. There was a high association between the two tests, with 
Table 2. - Association of polysomnography (PSG) results with overall investigator assessment of the video test in 58 children

\begin{tabular}{lccc}
\hline & \multicolumn{2}{c}{ Polysomnography } & \\
\cline { 2 - 3 } & Abnormal & Normal & Total \\
\hline Video test & & 7 & \\
$\quad$ Abnormal & 34 & 15 & 41 \\
$\quad$ Normal & 2 & 22 & 17 \\
\hline Total & 36 & & 58 \\
\hline
\end{tabular}

$\mathrm{p}<0.001$, Fisher's exact test.

an agreement in 49 of the 58 patients (84\%) (table 2). Disagreement was observed in 9 of the 58: in seven cases the video test was abnormal while the PSG was normal, and in two cases the PSG was abnormal while the video test was normal. The video test results reliably represented the PSG results (Kappa test of reliability $=0.65$; SE $=0.1$ ). Considering the PSG as the "gold standard", the overall interpretation of the video test yielded a sensitivity of $94 \%$ (34 out of 36) with a specificity of $68 \%$ (15 out of 22).

The distribution of the video test score by PSG results showed that all nine patients with scores $\leq 5$ had normal PSG, while all 23 patients with scores $\geq 11$ had abnormal PSG (fig. 1). Hence, cut-off points at scores 5 and 11 could predict PSG results in 55\% (32 out of 58).

Of the seven cases diagnosed as abnormal by overall investigator's judgement but with normal PSG, six had scores of 9-10 points. All cases considered to be abnormal by overall investigator's decision had scores $\geq 8$, and those considered as normals had scores $\leq 8$ points.

Applying the stepwise logistic regression, video parameters 1 and 7 (from table 1): "inspiratory noise" and "mouth breathing" were found to be nonsignificant as predictors of abnormal (or normal) PSG, and were therefore removed from the equation. The parameters: "type of inspiratory noise" and "chest retractions" were found to be the most significant (out of the five which were included in the stepwise logistic regression equation). Table 3 shows the coefficients of the five parameters. Applying these coefficients in the logistic regression

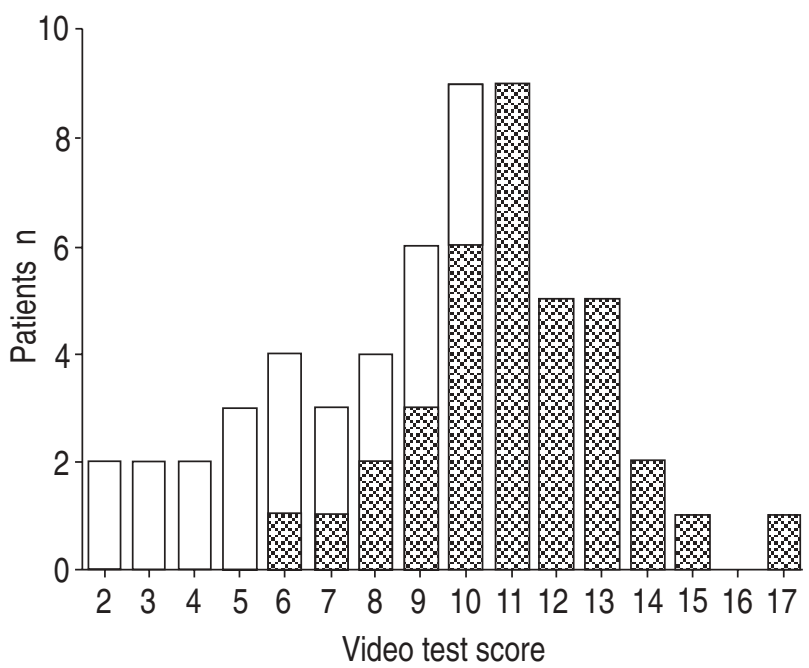

Fig. 1. - Distribution of video test score by polysomnography (PSG)

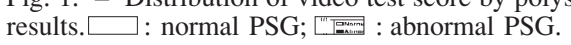

Table 3. - Results of logistic regression of video test scores evaluated against the polysomnography data

\begin{tabular}{lc}
\hline Parameter* & Coefficient $^{\#}$ \\
\hline Type of inspiratory noise & 31.08 \\
Movements during sleep & -16.33 \\
Number of waking episodes & 7.69 \\
Number of apnoeas: & \\
$\quad$ none (score=0) & 0 \\
when "1-2 episodes" (score=1) & -8.78 \\
$\quad$ when " $\geq 3$ episodes" (score=2) & 1.18 \\
Chest retractions: & 0 \\
$\quad$ none (score=0) & 25.69 \\
when "intermittent" (score=1) & 34.76 \\
$\quad$ when "all the time" (score=2) & -40.44 \\
Constant &
\end{tabular}

*: inspiratory noise and mouth breathing were excluded because they did not contribute to prediction; \#: the coefficients for the equation for: 1) the type of inspiratory noise; 2) movements during sleep; and 3) the number of waking episodes, are the same for all score values. The coefficients for number of apnoeas and for chest retractions depend on the results (score) for each parameter.

equation, the formulated equation was able to correctly predict 49 of the 58 patients (84\%) as follows: 32 out of 36 abnormals and 17 out of 22 normals. Probabilities close to zero $(\leq 0.3)$ predicted abnormal PSG, while probabilities close to one $(>0.7)$ predicted normal PSG. Nine cases were found to be borderline or inconclusive, i.e. confidence of prediction was low (probabilities ranged $0.3-0.7$ ). The sensitivity and specificity of the video score based on the logistic equation improved to $89 \%$ (32 out of 36) and 77\% (17 out of 22) respectively.

\section{Discussion}

The video test may add important information to the PSG. Obstructive episodes and abnormal PSGs have been found in up to $18 \%$ of normal (non-OSAS) children [9]. These children may not necessarily need treatment and clinical judgement is also required [9]. The best and probably the only way to obtain a reliable patient history and to perceive clinically the severity of the disorder in a child suspected of suffering from OSAS, is to observe sleep at home. The home sleep video test offers an easy and practical way to achieve this goal.

Several screening tests have been suggested. Daytime PSGs are inadequate to definitely exclude a diagnosis of OSAS [14-16]. BROUILLETTE and co-workers [10] suggested an OSA score as a screening method. Their score was based on only three clinical variables: 1) difficulty breathing during sleep; 2) apnoea; and 3) snoring, which were obtained from a questionnaire completed during parents' interview. The score was tested by comparison to daytime PSG and was successful in predicting only 3 out of 7 normal children and 8 out of 9 cases with OSAS. These authors also reported a subgroup of children with positive clinical symptoms but normal findings on PSG [10]. Preliminary data from other centres suggest that this method may not be applicable to all populations [17, $18]$. In another study of 65 snoring children with sleep disturbances, this clinical symptom score failed to reliably diagnose childhood OSAS [19]. Even an expanded clinical symptom score based on a questionnaire did not 
yield a predictive model for OSAS [17]. Our scoring system is based on seven variables which are assessed directly by the same experienced physicians watching the sleeping child. This may explain the much higher sensitivity and correlation of the video method observed in the present study. A consultant paediatrician was able to reach similar results even after a short period of training.

History alone grossly underestimates the incidence and severity of OSAS [7, 20, 21], therefore it has been suggested that parents should be asked to mimic their child's breathing pattern [4]. We believe that the videotape provides this information in a much more reliable and objective way. HAPONIK et al. [22] showed that bedside observation was a poor diagnostic tool for assessing OSA when compared with PSG in a group of 31 adults. The observation time in their study was limited to 5-10 min and no designated scoring system was used. The results of the present study show that a 30 min home sleep video test is in good agreement with polysomnography primarily when OSA is present (abnormal PSG).

Audiotapes have been used as screening tests. This technique is probably much inferior to videotapes because it monitors only one variable (noisy breathing/snoring).

STRADLING et al. [14] used $8 \mathrm{~h}$ video-recordings at home to monitor body movements in a group of 26 children with UAO due to adenotonsillar hypertrophy, and compared the results with simultaneous home oximetry. They showed that hypoxaemic dipping always corresponded to obstructed, or partially obstructed, noisy breathing. However, some children had sleep disturbance with body movements due to sleep apnoea or hypopnoea observed by the video-recordings without detectable hypoxaemia, presumably because arousal and reopening of the airway occurred before this could be detected [14]. These investigators used only one variable, the percentage time spent in moving. The present study shows that much more information can be obtained by the home video technique.

OSAS in children can be reliably diagnosed by meticulous observation of the sleeping child [14, 20, 23]. However, this requires that the patient sleeps at the medical centre or at the doctor's office, a situation that bears many of the disadvantages of PSG. Home video-recording during sleep overcomes this problem. Obviously, some data reduction is required before reviewing of an $8 \mathrm{~h}$ video-recording is practical [24]. The present study has shown that 30 min recordings of a representative sleep is an adequate alternative bearing a high rate of agreement in diagnosing and assessing OSAS in children. Studies comparing overnight simultaneous videorecording and PSG at home are needed. The first step has recently been carried out by BROUILLETTE and co-workers [25-26], who showed that a home cardiorespiratory recording system comprising respiratory inductance plethysmography, ECG and $\mathrm{Sa}, \mathrm{O}_{2}$ synchronized with videorecording, can replace full PSG in many cases. The video test is not only simple and highly economical, but also permits serial recordings and provides an easy way to follow the patients before as well as after treatment.

Predicting PSG results by the video test score is significantly enhanced by applying the stepwise logistic regression analysis (84\% compared to 55\%). It was interesting to observe that snoring and mouth breathing did not contribute to the diagnosis; the reason being that almost all children who were included in this study snored. This was, in fact, the main indication to include these children.

Another limitation of the video score is that it does not monitor sleep state. Hence, it is possible that because at least some of the video-recordings were not obtained during rapid eye movement (REM) sleep, the method missed cases which would be considered as abnormal if video-recording also included REM sleep.

The high sensitivity (abnormal video test with abnormal PSG) with a very low false-negative rate (normal video test with abnormal PSG) and the relatively low specificity (normal video test with normal PSG) make the video test an ideal tool to screen a large number of patients without the limitations of cost, patient inconvenience, and intrusive monitoring, thereby, decreasing the need for PSG. Children with a normal video test on overall interpretation and a score of $\leq 5$ points may be considered as normals. In these children, PSG is either not required or may be postponed. However, a repeat video test may be easily performed after some time when parents insist that the child still has any of the related symptoms. In children with an abnormal or borderline subjective video test and when the video score is 6-10 points a PSG is required. When the video score is $\geq 11$ the likelihood of OSAS is very high. Criteria based on the stepwise logistic regression improves selection of patients who need PSG.

In conclusion, home video-recording is a reliable screening test for obstructive sleep apnoea syndrome in children. Its use ameliorates patient selection for polysomnography. More studies comparing home video-recording to polysomnography are needed in order to improve the video score. This will probably increase the specificity of this tool.

Acknowledgements: The authors are grateful to P. Lilos from the Statistical Laboratory, Tel Aviv University for the statistical analyses.

\section{References}

1. American Thoracic Society. Standards and indications for cardiopulmonary sleep studies in children. Am J Respir Crit Care Med 1996; 153: 866-878.

2. Guilleminault C, Korobkin R, Winkle R. A review of 50 children with obstructive sleep apnea syndrome. Lung 1981; 159: 275-287.

3. Brouillette RT, Fernback SK, Hunt CE. Obstructive sleep apnea in infants and children. J Pediatr 1982; 100: 31-40.

4. Marcus CL, Carroll JL. Obstructive sleep apnea syndrome. In: Loughlin GM, Eigen H, eds. Respiratory Disease in Children. Diagnosis and Management. Baltimore, Maryland, Williams \& Wilkins, 1994; pp. 475-499.

5. Guilleminault C. Obstructive sleep apnea syndrome and its treatment in children: areas of agreement and controversy. Pediatr Pulmonol 1987; 3: 429-436.

6. Hunt CE, Brouillette RT. Disorders of breathing during sleep. In: Chernick V, Kendig EL Jr, eds. Disorders of the Respiratory Tract in Children. Philadelphia, Pennsylvania, W.B. Saunders, 1990; pp. 1004-1015.

7. Frank Y, Kravath RE, Pollack CP, Weitzman ED. Obstructive sleep apnea and its therapy: clinical and polysomnographic manifestations. Pediatrics 1983; 71: 737-742. 
8. Diagnostic Classification Steering Committee, Thorphy MJ, (Chairman). International classification of sleep disorders: diagnostic and coding manual. Rochester, Minnesota, American Sleep Disorders Association, 1990.

9. Marcus CL, Omlin KJ, Basinki DJ, et al. Normal polysomnographic values for children and adolescents. Am Rev Respir Dis 1992; 146: 1235-1239.

10. Brouillette RT, Hanson D, David R, et al. A diagnostic approach to suspected obstructive sleep apnea in children. J Pediatr 1984; 105: 10-14.

11. Rosen CL, D'Andrea L, Haddad GG. Adult criteria for obstructive sleep apnea do not identify children with serious obstruction. Am Rev Respir Dis 1992; 146: 1231-1234.

12. Gaultier C. Respiratory adaptation during sleep from the neonatal period to adolescence. In: Guilleminault C, ed. Sleep and Its Disorders in Children. New York, Raven Press, 1987; pp. 67-98.

13. BMDP Statistical Software. Dixon WJ (Chief Editor), University of California Press, 1990.

14. Stradling JR, Thomas G, Warley ARH, Williams P, Freeland A. Effect of adenotonsillectomy on nocturnal hypoxemia, sleep disturbance and symptoms in snoring children. Lancet 1990; 335: 249-253.

15. American Thoracic Society. Indications and standards for cardiopulmonary sleep studies. Am Rev Respir Dis 1989; 139: 559-568.

16. Marcus CL, Keens TG, Davidson Ward SL. Comparison of nap and overnight polysomnography in children. Pediatr Pulmonol 1992; 13: 16-21.

17. Carroll JL, McColley SA, Marcus CL, Curtis S, Pyzik P, Loughlin GM. Reported symptoms of childhood obstructive sleep apnea syndrome (OSA) vs primary snoring. Am Rev Respir Dis 1992; 145: A177.
18. Brouillette RT, Morielli A. Do all children suspected of obstructive sleep apnea (OSAS) need polysomnography (PSG) before tonsillectomy and adenoidectomy (T\&A)? Am J Respir Crit Care Med 1995; 151: A152.

19. Carroll JL, McColley SA, Marcus CL, Pyzik P, Curtis S, Loughlin GM. Can childhood obstructive sleep apnea syndrome (OSA) be diagnosed by clinical symptom score? Am Rev Respir Dis 1992; 145: A179.

20. Swift AC. Upper airway obstruction, sleep disturbance and adenotonsillectomy in children. J Laryngol Otol 1988; 102: 419-422.

21. Guilleminault C, Eldridge FL, Simmons FB, Dement WC. Sleep apnoea in eight children. Pediatrics 1976; 58: 23-30.

22. Haponik EF, Smith PL, Meyers DA, Bleecker ER. Evaluation of sleep-disordered breathing. Is polysomnography necessary? Am J Med 1984; 77: 671-677.

23. Potsic WP. Comparison of polysomnography and sonography for assessing regularity of respiration during sleep in adenotonsillar hypertrophy. Laryngoscope 1987; 97: 1430-1437.

24. Jacob SV, Morielli A, Mograss MA, Ducharme FM, Schloss MD, Brouillette RT. Home testing for pediatric obstructive sleep apnea syndrome secondary to adenotosillar hypertrophy. Pediatr Pulmonol 1995; 20: 241-252.

25. Brouillette RT, Jacob SV, Morielli A, et al. There's no place like home: evaluation of obstructive sleep apnea in the child's home. Pediatr Pulmonol 1995; 11(Suppl.): 86-88.

26. Mograss MA, Ducharme FM, Brouillette RT. Movement/ arousals. description classification and relationship to sleep apnea in children. Am J Respir Crit Care Med 1994; 150: 1690-1696. 